\title{
The SuperNEMO calorimeter
}

\section{Christine Marquet, on behalf of the SuperNEMO collaboration}

CENBG, Université de Bordeaux, CNRS/IN2P3, F-33175 Gradignan, France

E-mail: marquetecenbg.in2p3. fr

The SuperNEMO double beta decay project is a modular tracker-calorimeter experiment that will reach a sensitivity to the neutrinoless double beta decay half-life of $10^{26}$ years, corresponding to a Majorana neutrino mass of 50-100 meV. The main calorimeter is based on 440 Optical Modules made of large volume plastic scintillators $(10 \mathrm{~L})$ coupled with large area photomultipliers (Hamamatsu R5912-Mod). They are assembled in walls surrounding the isotope foil and the tracking volume. One of the main challenges of the SuperNEMO detector development programme was to reach an energy resolution, better than $8 \%(\mathrm{FWHM})$ at $1 \mathrm{MeV}$. The other challenge was to satisfy the radiopurity requirements for all the calorimeter materials. The calorimeter design of the first SuperNEMO module is presented as well as its performances and the status of its construction under way in the Modane underground laboratory.

38th International Conference on High Energy Physics 3-10 August 2016

Chicago, USA

${ }^{*}$ Speaker. 


\section{Introduction}

Neutrinoless double beta decay $(\beta \beta 0 v)$ is a sensitive probe of new physics beyond the Standard Model, establishing the Majorana nature of neutrinos. The half-life of the process $T_{1 / 2}^{0 v}$ is related to the neutrino physics parameters that describes the lepton number violation. In the easier case, where the $\beta \beta 0 v$ decay is due to the exchange of a light Majorana neutrino, this parameter is the effective neutrino mass $\left\langle m_{v}\right\rangle$ which would give information on the absolute neutrino mass scale and the mass hierarchy. The sensitivity to $T_{1 / 2}^{0 v}$ is determined by the detector performances as:

$$
T_{1 / 2}^{0 v}(A, Z)>\ln 2 \cdot \frac{N_{A}}{A} \cdot \frac{\varepsilon}{k_{C L}} \cdot \sqrt{\frac{M t}{N_{b k g} \Delta E}}
$$

where $N_{A}$ is the Avogadro number, A the atomic mass, $\varepsilon$ the $\beta \beta 0 v$ detection efficiency, $k_{C L}$ the confidence level, $\mathrm{M}$ the isotope mass, $\mathrm{t}$ the runtime, $N_{b k g}$ the background events (in $\mathrm{kg}^{-1} \mathrm{keV}^{-1}$ year ${ }^{-1}$ ) and $\Delta E$ the energy resolution of the $0 v \beta \beta$ decay at the $\mathrm{Q}$ value of the decay (in $\mathrm{keV}$ ). As the ultimate background to $\beta \beta 0 v$ is the irreducible $\beta \beta 2 v$ process, the energy resolution of the calorimeter is one of the main parameters determining the detector's overall sensitivity.

\section{The SuperNEMO detector}

The SuperNEMO detector design is based on the technology of the recently completed NEMO3 experiment [1], using a tracker-calorimeter detection technique. It is multi-observable detector that allows full topological reconstruction of events resulting in powerful background rejection. The SuperNEMO detector will hold $100 \mathrm{~kg}$ of ${ }^{82} \mathrm{Se}$ (baseline) or other isotopes like ${ }^{150} \mathrm{Nd}$ to reach a sensitivity of $\sim 10^{26}$ years to $T_{1 / 2}^{0 v}$, corresponding to a 50-100 meV effective Majorana neutrino mass [2]. It consists of 20 modules ( $4 \mathrm{~m} \times 6 \mathrm{~m} \times 2 \mathrm{~m}$ ) each containing $5 \mathrm{~kg}$ of ${ }^{82} \mathrm{Se}$ in the form of a thin $\left(\approx 40 \mathrm{mg} / \mathrm{cm}^{2}\right)$ vertically suspended source foil, surrounded by 2000 drift cells operating in Geiger mode for particle tracking and enclosed by calorimeter walls for energy and time of flight measurements (figure 1). A magnetic field of $25 \mathrm{G}$ allows to distinguish electrons from positrons. Passive shielding will surround the detector to reduce the environmental neutron and $\gamma$-ray background. The first SuperNEMO module, known as the Demonstrator, is currently under construction and is expected to start data taking in 2017.

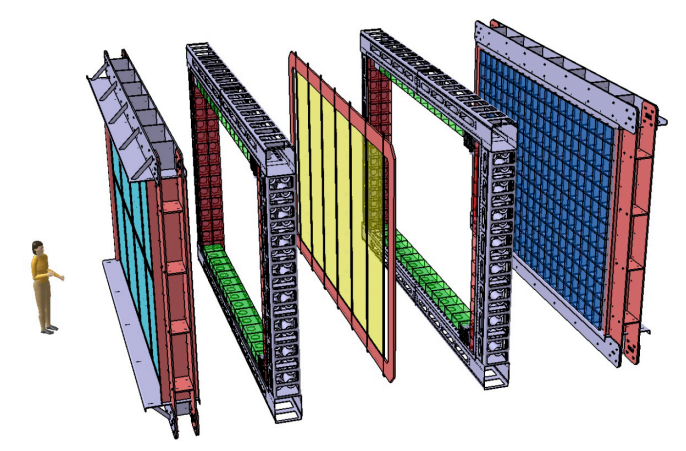

Figure 1: An exploded view of a SuperNEMO module, showing (from left to right) one calorimeter wall, one tracker volume, the source foil, another tracker volume and another calorimeter wall. 


\section{The calorimeter design and performances}

SuperNEMO has a need for a robust calorimeter using long-lasting technology that provides the required energy resolution of $8 \%$ (FWHM) for $1 \mathrm{MeV}$ electrons at a sensible cost and preserving the low background requirements. The geometry and material of the scintillator blocks have to be optimised for the detection of low energy $(\mathscr{O}(1 \mathrm{MeV}))$ electrons whilst providing efficient $\gamma$-ray tagging $(>50 \%$ at $1 \mathrm{MeV}$ ) for background suppression. The calorimeter R\&D for SuperNEMO consisted of three main areas of study: energy resolution, radiopurity and calibration. It has led to a main calorimeter consisting in 2 walls of optical modules (OM). The main $440 \mathrm{OMs}$ are square-faced $256 \mathrm{~mm} \times 256 \mathrm{~mm}$ scintillator blocks with a minimal thickness of $141 \mathrm{~mm}$ and a hemispherical 'cutout' directly coupled to an 8-inch photomultiplier (figure 2).
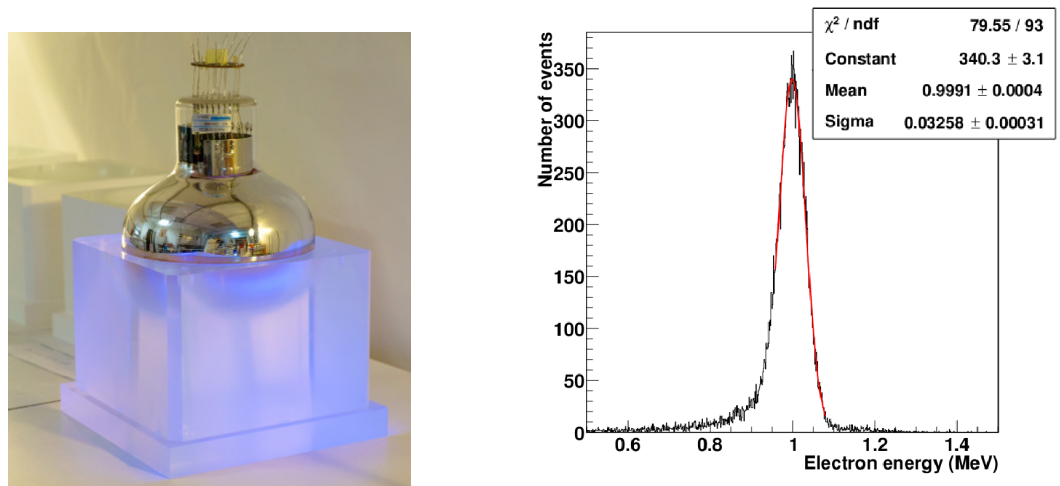

Figure 2: a: The SuperNEMO calorimeter optical module. b: Energy distribution of one SuperNEMO OM measured with $1 \mathrm{MeV}$ electrons (this distribution includes an additional $0.5 \% \Delta \mathrm{E} / \mathrm{E}$-in quadrature- caused by a coincidence trigger module used in the electron beam spectrometer

The scintillators are polystyrene (PS) plastic blocks produced by NUVIA whose composition and production procedures have been improved for SuperNEMO. The PMTs are low radioactive 8inch R5912-MOD PMT developed in collaboration with Hamamatsu. In order to increase the light collection, the blocks are wrapped in $600 \mu \mathrm{m}$ Teflon on the sides followed by $12 \mu \mathrm{m}$ aluminised Mylar on all the faces. The objectives of theR\&D have been achieved : energy resolution better than $8 \% / \sqrt{E}$ (FWHM) has been reached (figure 2), linearity is better than $1 \%$ below $Q_{\beta \beta}$, time resolution is $400 \mathrm{ps}(\sigma$ at $1 \mathrm{MeV})$ and all the materials of the OMs have been selected by germanium detectors for their low levels of radioactivity (dominated by the glass with $0.85 \mathrm{~Bq} / \mathrm{kg}$ for ${ }^{40} \mathrm{~K}$, $0.38 \mathrm{~Bq} / \mathrm{kg}$ for ${ }^{214} \mathrm{Bi}$ and $0.15 \mathrm{~Bq} / \mathrm{kg}$ for ${ }^{208} \mathrm{Tl}$ ). All the optical performances of the OMs have been characterised with two electron beam spectrometers providing mono-energetic (FWHM $1.0 \%$ at $1 \mathrm{MeV}$ ) electrons from 0.4 to $2.0 \mathrm{MeV}$ [3]. GEANT4-based optical simulations have been also developed for this $\mathrm{R} \& \mathrm{D}[4]$.

\section{The calorimeter production}

The production of the optical modules consists in the wrapping of the scintillators, in the optical coupling of the scintillators and PMTs and in the characterisation of each OM with the electron beam spectrometer to measure the energy resolution, linearity and spatial uniformity in 
terms of the impact point of the electrons on the entrance face of the block. $92 \%$ of OMs are produced. The mean energy resolution is $8 . \% 3$ with the first batches of scintillators (standard PS) and $8.0 \%$ after improvements on the scintillator production procedures (enhanced PS). The FWHM distributions are given in figure 3. The first wall of the calorimeter has been built in LSM. The second wall is under progress and will be finalised end of this year.

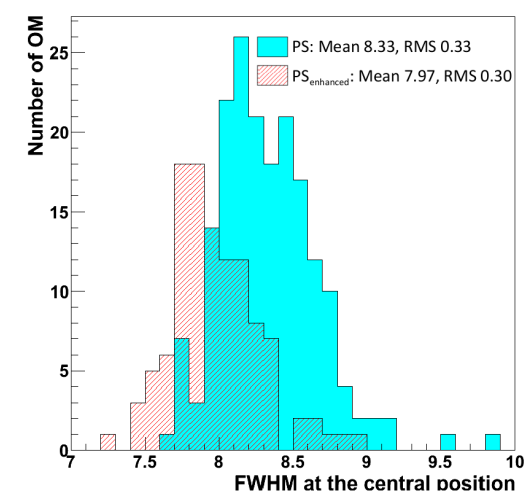

Figure 3: Distribution of the energy resolution (FWHM) measured with $1 \mathrm{MeV}$ electrons on the central position of the OM entrance face

\section{Conclusion}

One of the main focuses of the SuperNEMO R\&D has been on the calorimeter design, in particular improving the energy resolution, FWHM, for $1 \mathrm{MeV}$ electrons from (14-17)\% achieved by the previous NEMO-3 experiment to around 8\% for SuperNEMO using larger volume plastic scintillators without compromising other requirements such as the linearity, time resolution and radiopurity. Objectives have been reached and the construction of the calorimeter is now being finalised in the LSM underground laboratory.

\section{References}

[1] Arnold R. et al., Technical design and performance of the NEMO3 detector, Nucl. Instrum. Meth., A 536 (2005) 79.

[2] Arnold R. et al, Probing new physics models of neutrinoless double beta decay with SuperNEMO., Eur. Phys. J., C 70 (2010) 927.

[3] Marquet Ch. et al., High energy resolution electron beam spectrometer in the MeV range., 2015 JINST 10 P09008.

[4] Pahlka B. et al.,Spectral modelling of the scintillator for the NEMO-3 and SuperNEMO detectors., Nucl. Instrum. Meth., A 625 (2011) 20. 